\title{
COORDENADAS DE COR E CAROTENÓIDES TOTAIS EM POLPA DE GOIABA MICROENCAPSULADA OBTIDA POR SPRAY DRYER
}

\author{
L. A. L. MEWS ${ }^{1}$, R. CARMINATTI ${ }^{1}$, O. M. PORCU² e M. S. V. P. OVIEDO ${ }^{3}$ \\ ${ }^{1}$ Universidade Tecnológica Federal do Paraná - Campus Pato Branco, Programa Pós Graduação em \\ Processos Químicos e Bioquímicos - PPGTP. \\ ${ }^{2}$ Universidade Tecnológica Federal do Paraná - Campus Medianeira, SEBLIC - Química, Docente, \\ Programa Pós Graduação em Processos Químicos e Bioquímicos - Campus Pato Branco. \\ ${ }^{3}$ Universidade Tecnológica Federal do Paraná - Campus Campo Mourão, \\ Docente,Programa Pós Graduação em Tecnologia de Alimentos - PPGTA. \\ E-mail para contato: ornellamporcu@gmail.com
}

\begin{abstract}
RESUMO - A goiaba é uma fruta rica em carotenoides, em particular licopeno, carotenoide majoritário na goiaba, que apresenta comprovada ação contra o câncer de próstata. O objetivo deste estudo foi caracterizar a influência de dextrina nas características de cor e retenção de carotenoides totais na polpa de goiaba microencapsulada obtida por spray dryer. Dextrina foi adicionada a polpa de goiaba na proporção de 1:1 e homogeneizada até completa dissolução. A mistura obtida foi submetida ao processo de spray dryer e o pó resultante (MG) caracterizado quanto a atividade de água $(0,2617 \pm 0,0006), \mathrm{pH}(3,69 \pm 0,01),{ }^{0}$ Brix $(10,90 \pm 0,12)$, luminosidade $\left(\mathrm{L}^{*}=84,67 \pm 0,28\right)$, cromaticidade $\mathrm{a}^{*} \mathrm{e} \mathrm{b} *(7,28 \pm 0,48$ e $15,03 \pm 0,78$, respectivamente $)$ e carotenoides totais $(64,88 \pm 0,52 \mu \mathrm{g} / \mathrm{g})$. O elevado conteúdo de carotenoides totais e o perfil de cor desenvolvido por (MG) demonstrou que este suplemento é excelente matéria prima e pode servir como ingrediente funcional no desenvolvimento de novos produtos alimentícios.
\end{abstract}

\section{INTRODUÇÃO}

A goiaba é uma fruta tropical conhecida e caracterizada por baixo teor de carboidratos, gorduras, proteínas e alto teor de vitamina C (mais de $100 \mathrm{mg} / 100 \mathrm{~g}$ de fruta) e teor de fibra (2,8 $5,5 \mathrm{~g} / 100 \mathrm{~g}$ de frutas) (Gutiérrez et al., 2008). Ultimamente a goiaba tem chamado a atenção pelo alto teor de licopeno presente em sua composição. O licopeno é um carotenoide, com propriedades funcionais e com efeito comprovado contra o câncer de próstata (Porcu, 2014).

Os carotenoides possuem cadeia poliênica, constituída por um longo sistema de ligações duplas conjugadas, e devido a presença dessas insaturações sofrem facilmente oxidação, perda da cor, sensibilidade à luz, temperatura extrema e acidez (Silva et al., 2010). Dessa forma, a indústria alimentícia tem buscado novas alternativas para melhorar a estabilidade da cor e retenção de compostos bioativos tais como os carotenoides e, que apresentem baixo custo, como a 
microencapsulação em alimentos (Del Pozo-Insfran et al., 2004).

A microencapsulação em alimentos é o processo em que a matéria prima é cercada por revestimento comestível, obtendo-se pequenas cápsulas (Gharsallaoui et al., 2007; Dib Taxi et al., 2003). O uso da microencapsulação pela indústria de alimentos é proteger contra oxidação química os ingredientes encapsulados como pigmentos e compostos bioativos, tornando-os mais estáveis no que se diz respeito à qualidade nutricional e à cor. Como fatores da oxidação química temos os fatores do ambiente como temperatura, luz, pH e outros. (Barros e Stringheta, 2006; Clark, 2002; Depypere et al., 2003).

Alguns autores têm usado a microencapsulação para proteger compostos sensíveis como carotenoides e vitamina $\mathrm{C}$, em frutos como o tomate, acerola, goiaba, e ainda para aumentar a estabilidade do produto. Shu et al. (2006) utilizaram a microencapsulação para proteger o licopeno do tomate da instabilidade do ambiente e concluíram que o pó manteve-se estável durante o armazenamento. Patil et al. (2013) ao realizarem o spray dried da polpa da goiaba concluíram que o pó resultante possuía maior quantidade de vitamina $\mathrm{C}$ quando comparada à polpa comercializada. Esses autores chegaram à conclusão que a cor e a qualidade nutricional do microencapsulado resultante era elevada e estável.

Devido a importância de se obter produtos com características nutricionais de alto teor funcional agregado, o objetivo deste trabalho foi caracterizar a influência da dextrina nas coordenadas de cor, sólidos solúveis totais, atividade de água, e retenção de carotenoides totais na polpa de goiaba microencapsulada obtida por spray dryer.

\section{METODOLOGIA}

Esta pesquisa foi desenvolvida na Universidade Tecnológica Federal do Paraná, Campus Campo Mourão e Campus Pato Branco, no Laboratório de Panificação e de Bromatologia, respectivamente.

\subsection{Matéria prima}

Como matéria-prima da microencapsulação, foi utilizada polpa de goiaba designada por (G), gentilmente cedida pela Indústria de Polpas e Conservas Val Ltda., localizada no município de Vista Alegre do Alto (SP). A polpa foi armazenada em freezer $-10{ }^{\circ} \mathrm{C}$, sendo descongelada de acordo com a quantidade necessária para cada ensaio.

\subsection{Agente encapsulante}


Como agente encapsulante para realizar a microencapsulação da polpa de goiaba, foi utilizado o produto Dextrina 17 da empresa LORENZ®.

\subsection{Preparo da mistura}

Utilizou-se uma proporção de 1:1 de dextrina e polpa de goiaba na emulsão utilizada para microencapsulação. O preparo da mistura, denominada por Mix, a ser encapsulada consistiu em adicionar $210 \mathrm{~mL}$ de água destilada pré-aquecida a $80{ }^{\circ} \mathrm{C}$ à $41,5 \mathrm{~g}$ de dextrina, agitando-se em agitador mecânico de bancada (Fisatam 713D AAKER) a $4980 \mathrm{rpm} . \mathrm{min}^{-1}$ por 3 minutos para que a dextrina se dissolvesse na água. Foi adicionado à mistura anterior, $500 \mathrm{~g}$ de polpa de goiaba, voltando à agitação por mais 3 minutos a $1650 \mathrm{rpm} \cdot \mathrm{min}^{-1}$.

\subsection{Spray Dryer}

A secagem por atomização foi realizada em um secador laboratorial com sistema de atomização em Spray Dryer - Lab Maq modelo MSD 1.0. O fluxo de ar comprimido para secagem de 45 L.min ${ }^{-1}$, temperatura de secagem ajustada em $120{ }^{\circ} \mathrm{C}$ e a velocidade de secagem de $0,54 \mathrm{~L}_{\mathrm{h}}{ }^{-1}$. A temperatura de saída do produto também foi monitorada, variando de 63,6 a $66,0^{\circ} \mathrm{C}$. Depois do processo de pulverização, o pó foi recolhido num recipiente de vidro âmbar com fechamento hermético. $\mathrm{O}$ microencapsulado de polpa de goiaba obtido foi denominado (MG).

\section{$2.5 \mathrm{pH}$}

Para determinar o $\mathrm{pH}$ de $(\mathrm{MG})$ utilizaram-se $2 \mathrm{~g}$ de amostra, na qual foram acrescentadas 10 $\mathrm{mL}$ de água destilada. Homogeneizou-se a mistura e deixou-se em repouso por 10 minutos. $\mathrm{O} \mathrm{pH}$ foi medido diretamente no sobrenadante (Instituto..., 2008).

\subsection{Atividade de água (Aw)}

A leitura de atividade de água (Aw) foi feita para (G) e (MG) em triplicata. Esta análise foi realizada em aparelho específico para esta determinação (marca Novasina).

\subsection{Sólidos Solúveis Totais}

Para a polpa de goiaba (G) e Mix (água + polpa de goiaba + dextrina), esta determinação foi feita através de um refratômetro (Instrutherm, RT-10ATC). Utilizaram-se 3 a 4 gotas da polpa e da emulsão para medida no prisma do refratômetro, realizada em triplicata. A leitura foi feita pela escala de graus Brix (Instituto..., 2008).

\subsection{Carotenoides Totais}

A metodologia utilizada foi a descrita por Porcu (2004), onde o teor de carotenoides totais é baseado na quantificação do carotenoide majoritário presente, o licopeno.

O teor de carotenoides totais, baseado em licopeno, calcula-se pela Equação 1: 


$$
C T=\frac{A_{470 n m} \times V \times 10^{4}}{A_{1 \mathrm{~cm}}^{1 \%} \times m}
$$

Onde:

$\mathrm{A}_{\mathrm{nm}}=$ absorbância máxima lida no espectro;

$\mathrm{A} \frac{1 \%}{1 \mathrm{~cm}}=$ coeficiente de absorção molar (éter de petróleo $=3452$ )

$\mathrm{V}=$ volume da diluição do extrato $(\mathrm{mL})$;

$\mathrm{m}=$ massa da amostra $(\mathrm{g})$;

$\mathrm{CT}=$ teor de carotenoides totais $(\mu \mathrm{g} / \mathrm{g})$

\subsection{Cor}

A cor do mix e do microencapsulado de polpa de goiaba, foi determinada utilizando-se o colorímetro Hunter Lab Mini ScanEZ. Os resultados foram interpretados pelo sistema CIELAB, utilizando as coordenadas $\mathrm{L}^{*}, \mathrm{a}^{*} \mathrm{e} \mathrm{b}^{*}$. Os parâmetros de cor tais como chroma $\left(\mathrm{Cab}^{*}\right)$ e tonalidade $\left(h^{*}{ }_{a b}\right)$ foram calculados de acordo com as Equações 2 e 3, respectivamente.

$$
\begin{aligned}
& C_{a b}^{*}=\left[\left(a^{*}\right)^{2}+\left(b^{*}\right)^{2}\right]^{1 / 2} \\
& h_{a b}^{*}=\arctan \left(\frac{b^{*}}{a^{*}}\right)
\end{aligned}
$$

\subsection{Análises Estatísticas}

As análises foram realizadas em triplicata e os resultados foram analisados estatisticamente através de Teste de Tukey e Teste t, a 5 \% de significância $(\mathrm{p} \leq 0,05)$, com o auxílio do software R.

\section{RESULTADOS E DISCUSSÕES}

Nos estudos de coordenadas de cor L* representa a luminosidade numa escala de 0 (preto) a 100 (branco); $a^{*}$ representa a variação das tonalidades das cores verde e vermelho, onde valores positivos de $\mathrm{a}^{*}$ correspondem a intensidade da cor vermelha e valores negativos correspondem à intensidade da cor verde; $b^{*}$ representa a variação das tonalidades das cores azul e amarelo, onde valores positivos de $b^{*}$ correspondem a intensidade da cor amarela enquanto que valores negativos correspondem à intensidade da cor azul. A coordenada $C^{*}$ (Chroma) representa o grau de concentração ou pureza de 


\section{9 a 22 de outubro de 2014 \\ Florianópolis/SC}

uma cor e o h* ${ }_{a b}$ representa a tonalidade das cores. Em relação ao comportamento da pureza da cor, quando $\mathrm{h}^{*}{ }_{a b}$ é $0^{\circ}$ é fixado no eixo horizontal tende-se a (+) (vermelho) e, girando no sentido antihorário na sequência, quando $\mathrm{h}_{a b}$ sendo igual a $90^{\circ}$ tende-se ao amarelo, com $\mathrm{h}^{*}{ }_{a b}=180^{\circ}$ tende-se ao verde e $\mathrm{h}^{*}{ }_{a b}=270^{\circ}$ tende-se ao azul (Alves et al., 2008).

A polpa de goiaba apresentou um aumento da luminosidade ( $\left.\mathrm{L}^{*}\right)$ ao ser misturada ao agente encapsulante (dextrina) e após sofrer o processo no spray dryer a luminosidade do pó resultante tendeu em direção ao branco apresentando valores de 84,67 0,28 conforme verificamos na Tabela 1 .

Tonon et al. (2009) ao microencapsularem suco de açaí utilizando como agente encapsulante a maltodextrina observaram aumento do parâmetro luminosidade $\left(\mathrm{L}^{*}\right)$ para o pó resultante do suco de açaí. Segundo os autores, o aumento do parâmetro $L^{*}$ já era esperado, pois a maltodextrina apresenta cor branca e, dessa forma, dilui a coloração roxa característica do suco de açaí. O mesmo foi verificado neste estudo após a adição de dextrina que diluiu a cor da polpa de goiaba levando-se posteriormente a cor rosácea do produto obtido (MG).

Tabela 1 - Parâmetros médios de cor e de carotenoides totais resultantes para (G), Mix e (MG)

\begin{tabular}{|c|c|c|c|c|c|c|}
\hline \multirow{2}{*}{ Amostra } & \multicolumn{5}{|c|}{ Cor } & \multirow{2}{*}{$\begin{array}{l}\text { Carotenoides } \\
\text { Totais }(\mu \mathrm{g} / \mathrm{g})\end{array}$} \\
\hline & $\mathbf{L}^{*}$ & $\mathbf{a}^{*}$ & $\mathbf{b}^{*}$ & C* ${ }_{a b}$ & $\mathbf{H}^{*}{ }_{a b}$ & \\
\hline (G) & $40,75^{\mathrm{a}} \pm 0,09$ & $16,32^{\mathrm{a}} \pm 0,07$ & $18,13^{\mathrm{a}} \pm 0,09$ & $24,39^{\mathrm{a}} \pm 0,11$ & $48,01^{\mathrm{a}} \pm 0,06$ & $32,62^{a} \pm 4,17$ \\
\hline Mix & $44,49^{b} \pm 0,14$ & $13,61^{\mathrm{b}} \pm 0,05$ & $14,19^{\mathrm{b}} \pm 0,08$ & $19,66^{\mathrm{b}} \pm 0,09$ & $46,21^{\mathrm{b}} \pm 0,05$ & $23,32^{a} \pm 3,62$ \\
\hline (MG) & $84,67^{\mathrm{c}} \pm 0,28$ & $7,28^{\mathrm{c}} \pm 0,48$ & $15,03^{\mathrm{b}} \pm 0,78$ & $16,82^{\mathrm{c}} \pm 0,91$ & $63,29^{c} \pm 0,32$ & $64,88^{b} \pm 0,52$ \\
\hline
\end{tabular}

Letras diferentes na mesma coluna indicam diferença significativa $(\mathrm{p}<0,05)$ entre os resultados obtidos para polpa de goiaba (G), Mix (mistura) e microencapsulado de polpa de goiaba (MG).

Os valores de cromaticidade para coordenada $b^{*}$ não apresentaram diferença significativa entre o mix $(14,19 \pm 0,08)$ e o microencapsulado de goiaba $(\mathrm{MG})(15,03 \pm 0,78)$. No entanto, a polpa de goiaba industrializada apresentou valores superiores $(16,32 \pm 0,07)$ ao mix e ao MG para essa coordenada (Tabela 1). Valores similares aos encontrados por Tonom et al. (2009) que ao microencapsularem suco de açaí não encontraram diferenças significativas entre o mix de açaí e o pó resultante do açaí, mas a diferença foi significativa para o suco de açaí puro.

As cromaticidades para coordenada $a^{*}$ sofreram um decréscimo significativo na tonalidade

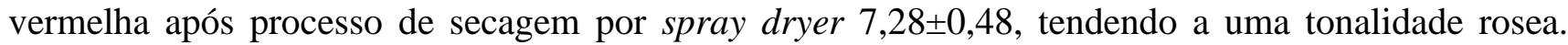
Segundo vários autores Tonom et al. (2009), Osorio et al, (2011), Quek et al. (2006), a adição do agente carreador pode provocar uma diminuição do parâmetro $\mathrm{a}^{*}$, ou seja, uma redução na tonalidade vermelha, também relacionada à diluição da cor, provocada pela adição deste agente carreador.

No presente estudo a coordenada $a^{*}$ diminuiu quando a amostra foi microencapulada, no entanto o parâmetro b* não apresentou diferença significativa entre MG e a mistura (Tabela 1). 


\section{9 a 22 de outubro de 2014 \\ Florianópolis/SC}

Segundo Quek et al. (2006), este fato pode ter contribuído para a mudança de valores no ângulo $\mathrm{h}^{*}{ }_{a b}$ e no Croma $\mathrm{C}^{*}{ }_{a b}$. Com a temperatura de entrada elevada, a cor do pó tornou-se mais escura. Uma das explicações para esse fato é que a goiaba apresenta alto teor de sólidos solúveis, o que pode ter contribuído para o escurecimento do MG.

O teor de carotenoides para o MG foi significativamente maior quando comparado a mistura e à (G), como é apresentado na Tabela 1. Tonom et al. (2009) e Quek et al. (2006), ao avaliarem compostos bioativos (antocianinas e licopeno) em microencapsulado do suco de açaí e na melancia encontraram quantidade superior no suco de açaí e na fruta, e menor quantidade nos microencapsulados, resultados contrários ao presente estudo. Os autores atribuíram esses resultados a alta temperatura de saída que levou às maiores perdas de antocianinas e licopeno, pois esses pigmentos possuem alta sensibilidade a temperaturas muito elevadas.

Neste estudo, a temperatura de secagem foi $120{ }^{\circ} \mathrm{C}$ e a temperatura de saída foi $60{ }^{\circ} \mathrm{C}$. Estas resultaram inferiores aos estudos relatados para o encapsulamento de outros carotenóides com diferentes matrizes alimentares tais como bixina (Barbosa e Mercadante, 2006). Por outro lado, o encapsulamento do suco de pitanga roxa resultou em maior teor de carotenóides tais como o licopeno, o $\beta$-caroteno, zeaxantina e luteína (Rutz, 2013). Este fato também foi verificado em nossa pesquisa. O aumento do teor de carotenóides totais no MG pode ser explicado devido ao tipo de revestimento seleciondo, dextrina utilizada na proporção de 1:1, a qual parece ter sido eficaz contra a oxidação dos carotenoides totais ocasionada pela baixa temperatura de saída do equipamento. E, ainda a matriz alimentar utilizada, polpa de goiaba, pode ter influenciado na manutenção do teor de carotenoides totais nas microcápsulas.

Assim, é provável que o tipo de resvestimento, ou seja, o agente encapsulante selecionado (dextrina) e o grupo de compostos encontrados originalmente na matriz alimentar (polpa de goiaba) além dos carotenoides, influenciaram os resultados e a eficiência da encapsulação.

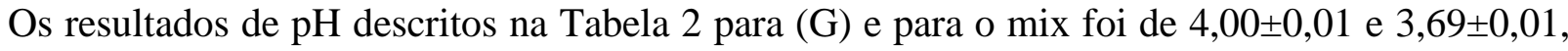
respectivamente, tendo apresentado diferença significativa entre si. Os valores médios de sólidos solúveis da polpa de goiaba e da mistura apresentaram diferença significativa, demonstrando aumento de sólidos solúveis devido a adição de dextrina.

Tabela 2 - Valores médios de sólidos solúveis expressos em graus Brix $\left({ }^{\circ} \mathrm{B}\right)$ e pH para (G) e Mix

$\begin{array}{lll}\text { Análise } & (\mathrm{G}) & \text { Mix }\end{array}$

Sólidos solúveis $\left({ }^{\circ} \mathrm{B}\right)$

$$
\begin{array}{lr}
8,30 \pm 0,00 * * & 10,90 \pm 0,12^{* *} \\
4,00 \pm 0,01 * * & 3,69 \pm 0,01 * *
\end{array}
$$$$
\mathrm{pH}
$$

(**) na mesma linha indicam diferença significativa $(\mathrm{t}<0,01)$ entre os resultados obtidos da polpa da goiaba $(\mathrm{G})$ e mix (dextrina e polpa de goiaba) 
O valor médio da atividade de água encontrada nesse estudo foi de 0,2617 $\pm 0,0006$ para MG, dado presente na Tabela 3, e esse valor está conforme o encontrado em outros estudos realizados em microencapsulados (Osorio et al., 2011). Quek et al. (2007) ao realizar a secagem de suco de melancia adicionado de maltodextrina por spray dryer encontrou valores de atividade de água que variaram de 0,2000 a 0,2900, os autores atribuíram a baixa atividade de água do microencapsulado a maltodextrina utilizada como agente carreador. Sendo assim, fica evidente que no presente estudo, a proporção de dextrina utilizada auxiliou na diminuição da atividade de água do (MG).

Tabela 3 - Valores médios de atividade de água (Aw) para (G) e (MG)

Amostra

Aw

$$
0,9900 \pm 0,0000 * *
$$

(MG)

$0,2617 \pm 0,0006^{* *}$

(**) na mesma linha indicam diferença significativa $(\mathrm{t}<0,01)$ entre os resultados obtidos da polpa da goiaba $(\mathrm{G})$ e a polpa de goiaba microencapsulada (MG)

\section{CONCLUSÃO}

O elevado conteúdo de carotenoides totais e o perfil de cor desenvolvido por (MG) demonstrou que este suplemento é excelente matéria prima e pode servir como ingrediente funcional no desenvolvimento de novos produtos alimentícios.

\section{REFERÊNCIAS}

ALVES, C. C. O.; RESENDE, J. V.; CRUVINEL, R. S. R; PRADO, M.E.T. Estabilidade da microestrutura e do teor de carotenóides de pós obtidos da polpa de pequi (Caryocar brasiliense Camb.) liofilizada .Ciên. e Tecnol. de Alimentos,v.28, p. 830-839, 2008.

BARBOSA, I. M. J.; MERCADANTE, A. Z. Avaliação da estabilidade das microcápsulas de bixina em diferentes matrizes alimentares Tecnol. \& Ciên. Agropec., João Pessoa, v.2, n.1, p.23-26, mar. 2008.

BARROS, F. A. R.; STRINGHETA, P. C. Microencapsulamento de Antocianinas: uma alternativa para o aumento de sua aplicabilidade com ingrediente alimentício. Biotecnol. Ciên. \& Desenvolvimento, v. 36, p. 18-24, 2006. 
CLARK, J. P. Food encapsulation:capturing one substance by another. Food Technol., v.56, p.63-65, 2002.

DEL POZO-INSFRAN, D.; BRENES, C. H.; TALCOTT, S. T. Phytochemical composition and pigment stability of Acai (Euterpe oleracea Mart.). J. Agric Food Chem. v. 52, n.6, p. 1539-45, 2004.

DEPYPERE, F.; DEWETTINCK, K.; RONSSE, F.; PIETERS, J. G. Food poder microencapsulation: principles, problems and oportunities. App. Biotecn., Food Science and Policy, v.1, p.75-94, 2003.

GHARSALLAOURI, A.; ROUDART, G.; CHAMBIN, O.; VOILLEY, A.; SAUREL, R. Applications of spray-drying in microencapsulation of food ingredients: An overview. Food Res. Int., v. 40, p. 1107-1121, 2007.

GUTIÉRREZ, R. M. P.; MITCHELL, S., SOLIS, R. V. Psidium guajava: uma revisão de seu uso tradicional, fitoquímica e farmacologia. J. Ethnopharmacol, v.17, p.1-27, 2008

INSTITUTO Adolfo Lutz. Métodos Físico-químicos para Análise de Alimentos. 4 ed. São Paulo: Instituto Adolfo Lutz, 2008.

OLIVEIRA, M. I. S.; TONON, R. V.; NOGUEIRA, R. V.; CABRAL, L. M. C. Estabilidade da polpa de morango atomizada utilizando diferentes agentes carreadores. Braz. J. Food Technol, Campinas, v.16, n.4, p. 310-18, out./dez., 2013.

OSORIO, C.; FORERO, D. P.; CARRIAZO, J. G. Characterisation and performance assessment of guava (Psidium guajava L.) microencapsulates obtained by spray-drying. Food Res. Int. v.44, p.1174-1181, 2011.

PATIL V.; CHAUHANA, A.K.; SINGHB, R.P. Optimization of the spray-drying process for developing guava poder using response surface methodology. Powder Technol., v.253, p. 230 236, 2014.

PORCU, O. M. Fatores que influenciam na composição de carotenoides em goiaba, acerola, pitanga e seus produtos processados. 2004. 131 f. Tese. (Doutorado em Ciência de Alimentos) Faculdade de Engenharia de Alimentos da Universidade Estadual de Campinas, Campinas, 2004.

PORCU, O. M. Desenvolvimento de Produtos alimentícios com valor funcional agregado: uma alternativa agroindustrial ISBN: 978-85-67 130-07-1 Lorena: Editôra Cabedalis, São Paulo, 2014 (in press).

QUEK, S. J.; CHOK, N. R.; SWEDLUND, P. The physicochemical properties of spray-dried watermelon powders. Chem. Eng.and Proces.: Proc. Intensification, v.46, mai., p. 386-392, 2007. 
RUTZ, Josiane Kuhn Caracterização e Encapsulação do Suco de Pitanga Roxa (Eugenia uniflora L). Dissertação (Mestrado em Ciência e Tecnologia de Alimentos) - Universidade Federal de Pelotas, Pelotas, 2013.

SILVA, M. L. C.; COSTA, R. S.; SANTANA, A. dos S.; KOBLITZ, M. G. B. Compostos fenólicos, carotenóides e atividade antioxidante em produtos vegetais. Semina: Ciên. Agrárias, Londrina, v. 31, n. 3, 2010.

SHU, B.; YU, W.; ZHAO Y., LIU, X. Study on microencapsulation of lycopene by spray-drying. Journal of Food Engineering, v.76, p.664-669, 2006.

TONON, R.V.; BRAB, C.; HUBINGER, M.D. Influência da temperatura do ar de secagem e da concentração de agente carreador sobre as propriedades físico-químicas do suco de açaí em pó. Ciênc. Tecnol. Aliment. Campinas, v. 29(2): p. 444-450, abr.-jun., 2009.

XAVIER, D. Suplementação da Farinha de Trigo Integral com Ingredientes Funcionais Aplicada ao Desenvolvimento de Produto Alimentício para Diabéticos Insulino não Dependentes. Dissertação (Mestrado em Processos Químicos e Bioquímicos) - Universidade Tecnológica Federal do Paraná, Pato Branco, 2013. 